University of New Orleans

ScholarWorks@UNO

$5-1-1984$

\title{
Division-of-wave-front polarizing beam splitter and half-shade device using dielectric thin film on dielectric substrate
}

R. M.A. Azzam

University of New Orleans, razzam@uno.edu

Follow this and additional works at: https://scholarworks.uno.edu/ee_facpubs

Part of the Electrical and Electronics Commons, and the Optics Commons

\section{Recommended Citation}

R. M. A. Azzam, "Division-of-wave-front polarizing beam splitter and half-shade device using dielectric thin film on dielectric substrate," Appl. Opt. 23, 1296-1298 (1984)

This Article is brought to you for free and open access by the Department of Electrical Engineering at ScholarWorks@UNO. It has been accepted for inclusion in Electrical Engineering Faculty Publications by an authorized administrator of ScholarWorks@UNO. For more information, please contact scholarworks@uno.edu. 
This section was established to reduce the lead time for the publication of Letters containing new, significant material in rapidly advancing areas of optics judged. compelling in their timeliness. The author of such a Letter should have his manuscript reviewed by an OSA Fellow who has similar technical interests and is not a member of the author's institution. The Letter should then be submitted to the Editor, accompanied by a LETTER OF ENDORSE
MENT FROM THE OSA FELLOW (who in effect has served as the referee and whose sponsorship will be indicated in the published Letter), A COMMITMENT FROM THE AUTHOR'S INSTITUTION TO PAY THE PUBLICATION CHARGES, and the signed COPYRIGHT TRANSFER AGREEMENT. The Letter will be published without further refereeing. The latest Directory of OSA Members, including Fellows, is published in the May/June 1982 issue of Optics News.

\section{Division-of-wave-front polarizing beam splitter and half-shade device using dielectric thin film on dielectric substrate}

\section{R. M. A. Azzam}

University of New Orleans, Department of Electrical Engineering, Lakefront, New Orleans, Louisiana 70148.

New Orleans, Louisiana 70148.

Received 2 March 1984.

Sponsored by Jean M. Bennett, Naval Weapons Center. 0003-6935/84/091296-03\$02.00/0.

(C) Optical Society of America.

A novel device is described that can be used to generate, by reflection, polarized optical wave fronts of juxtaposed fields of alternating orthogonal linear polarization states. It simply consists of a dielectric ${ }^{1}$ substrate partially covered by a transparent thin film of certain refractive index and thickness, with the (e.g., lithographically obtained) pattern of coated and uncoated areas corresponding to the desired polarization pattern of the reflected wave. A plane wave is incident at the Brewster angle of the uncoated substrate, and this suppresses the parallel $p$ electric-field vibration of the wave reflected from the uncoated areas. The film refractive index and thickness are selected to suppress, at the same angle, the perpendicular $s$ electric-field vibration of the wave reflected from the coated areas. Thus we achieve integrated crossed thin-film reflection polarizers (ICTFRP) on the same substrate. We also achieve, for the time, what we may call a division-of-wave-front polarizing beam splitter (DOWPBS) and the thin-film reflective half-shade device (TFRHSD). ${ }^{2}$ Important applications are anticipated, particularly for beam shaping, ellipsometry, and metrology.

Operation of this device can be readily explained with reference to Fig. 1. A plane wave is incident at angle $\phi$ in a medium of refractive index $N_{0}$ on a dielectric substrate of refractive index $N_{2}$ partially covered by a dielectric thin film of refractive index $N_{1}$ and thickness $d$. Normalized refractive indices are defined as

$$
n_{1}=N_{1} / N_{0}, \quad n_{2}=N_{2} / N_{0}
$$

To suppress the $p$-polarized component of light reflected from the uncoated substrate, incidence at the Brewster angle is required:

$$
\tan \phi_{p}=n_{2}
$$

The complex-amplitude reflection coefficient of the filmsubstrate system is given by ${ }^{3}$

$$
R_{\nu}=\left[r_{01 \nu}+r_{12 \nu} \exp (-j 2 \pi \zeta)\right] /\left[1+r_{01 \nu} r_{12 \nu} \exp (-j 2 \pi \zeta)\right],
$$

where $r_{01 \nu}$ and $r_{12 \nu}$ are the Fresnel reflection coefficients of the 01 and 12 interfaces for the $\nu(=p$ or $s)$ polarization, and $\zeta$ is the normalized film thickness

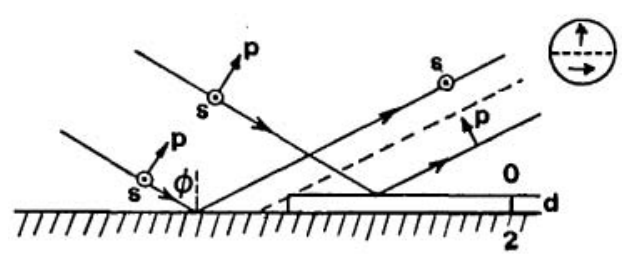

Fig. 1. Division-of-wave-front polarizing beam splitter and halfshade device consisting of a dielectric substrate (medium 2) partially covered by a dielectric thin film (medium 1) of appropriate refractive index and thickness $d . \quad p$ and $s$ indicate the linear polarizations parallel and perpendicular to the plane of incidence, respectively, of

a plane wave incident and reflected at angle $\phi$ in medium 0 .

$$
\begin{gathered}
\zeta=d / D_{\phi \cdot} \\
D_{\phi}=\frac{\lambda}{2 N_{0}}\left(n_{1}^{2}-\sin ^{2} \phi\right)^{-1 / 2}
\end{gathered}
$$

is the film thickness period, and $\lambda$ is the free-space wavelength of light. Extinction of the $s$ polarization upon reflection occurs when

$$
\begin{gathered}
r_{01 s}=r_{12 s}, \\
\zeta=1 / 2,
\end{gathered}
$$

as can be verified by direct substitution into Eq. (3). Equation (6a) leads to

$$
S_{1}^{2}=S_{0} S_{2}
$$

where

$$
S_{i}=\left(N_{i}^{2}-N_{0}^{2} \sin ^{2} \phi\right)^{1 / 2}, \quad i=0,1,2 .
$$

The antireflection condition for the $s$ polarization at oblique incidence, Eq. (7a), takes the same form as, and reduces to, the familiar antireflection condition $N_{1}^{2}=N_{0} N_{2}$ at normal incidence $(\phi=0)$. Equations (7) can be solved for the $s$ suppressing polarizing angle $\phi_{s}$ of the coated substrate:

$$
\tan ^{2} \phi_{s}=\frac{n_{2}^{2}-n_{1}^{4}}{\left(1-n_{1}^{2}\right)^{2}} .
$$

For operation as DOWPBS and TFRHSD the $p$ - and $s$ polarizing angles must be equal, i.e.,

$$
\phi_{p}=\phi_{s}=\phi .
$$

From Eqs. (2), (8), and (9) we obtain

$$
n_{1}^{2}=\frac{2 n_{2}^{2}}{\mathrm{n}_{2}^{2}+1}
$$

or

$$
n_{1}=\frac{\sqrt{ } 2}{\left[1+\left(1 / n_{2}^{2}\right)\right]^{1 / 2}} .
$$




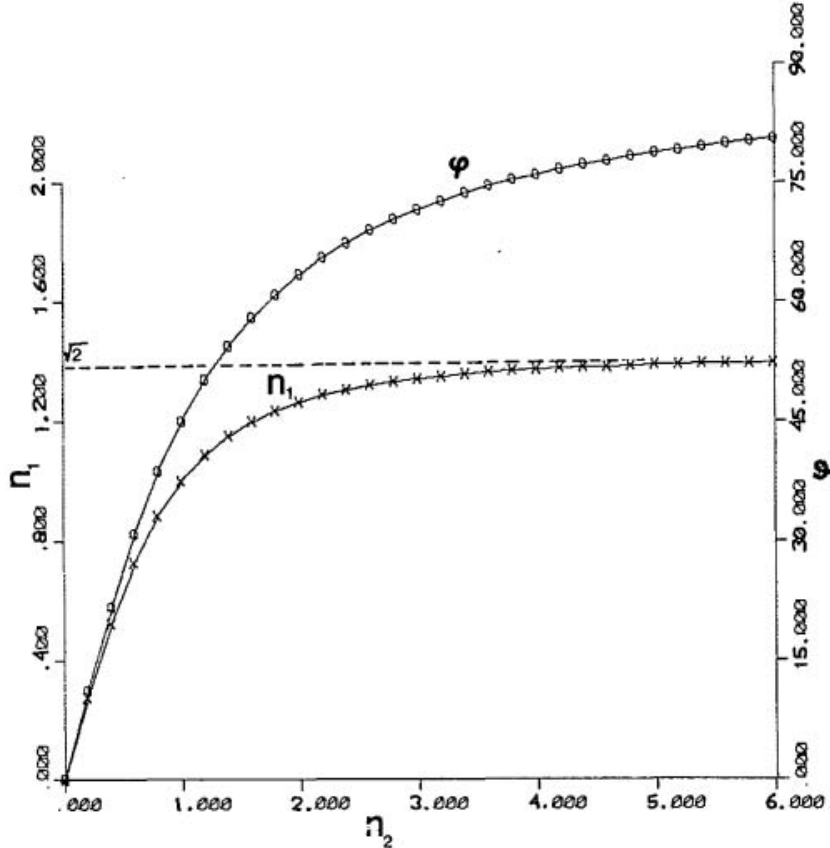

Fig. 2. Polarizing angle of incidence $\phi$ [Eq. (2)] and film refractive index $n_{1}$ [Eq. (10b)] vs substrate refractive index $n_{2} . n_{2}<1$ implies a dense medium of incidence and internal reflection.

Equation (10a) or (10b) determines the required film refractive index for given refractive indices of the substrate and medium of incidence. If the ambient is air (or vacuum), as often is the case, Eq. (10b) indicates that the film refractive index must be $<\sqrt{ } 2 \simeq 1.414$. Fluoride film materials (such as cryolite, chiolite, $\mathrm{LiF}, \mathrm{MgF}_{2}, \mathrm{CaF}_{2}$, and $\mathrm{ThF}_{4}$ ) do have this low refractive index. ${ }^{4}$ (For example, $\mathrm{CaF}_{2}$ can be prepared by vacuum evaporation with refractive indices in the 1.23-1.46 range. ${ }^{5}$

Figure 2 shows the film refractive index and common polarizing angle as functions of the substrate refractive index.

The required film thickness $d$ is equal to one (or an odd integral multiple of) half of the film thickness period evaluated at the Brewster angle. From Eqs. (4), (5), and (6b), we obtain the minimum required thickness:

$$
d_{s}=\sqrt{2}\left(\lambda / 4 N_{1}\right),
$$

i.e., $d_{s}=$ a certain fraction $(\sqrt{2} / 4=0.3536)$ of the wavelength of light in the film material.

The unextinguished $s$ reflection coefficient of the uncoated substrate at the Brewster angle is given by ${ }^{6}$

$$
R_{s}=\cos 2 \phi .
$$

The unextinguished $p$ reflection coefficient of the coated substrate at the same Brewster angle is obtained from Eq. (3), after some manipulations, as

$$
R_{p}=2 r_{01 p} /\left(1+r_{01 p}^{2}\right)
$$

or

$$
R_{p}=-\cos ^{2} 2 \phi /\left(2-\cos ^{2} 2 \phi\right) .
$$

From Eqs. (12) and (13b), we get the following relation between the unextinguished reflection coefficients of the coated and uncoated areas of the substrate:

$$
R_{p}=-R_{s}^{2} /\left(2-R_{s}^{2}\right) .
$$

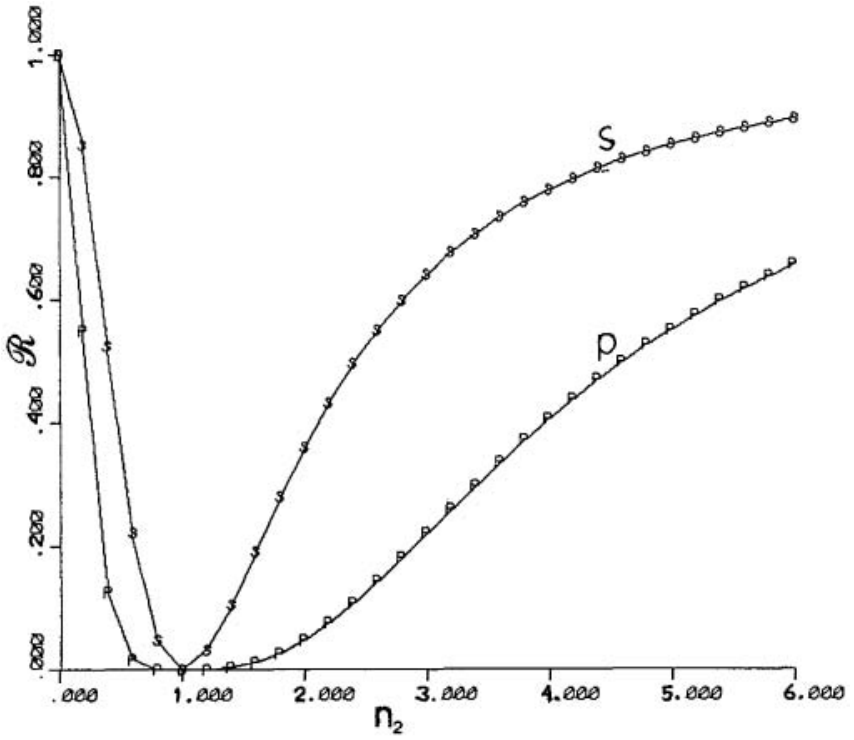

Fig. 3. Unextinguished $s$ and $p$ intensity reflectances $\mathcal{R}_{s}$ and $\mathscr{R}_{p}$ of the uncoated and coated areas, respectively, vs the substrate refractive index $n_{2}$.

The corresponding intensity reflectances $\left(\mathcal{R}=|R|^{2}\right)$ are interrelated by

$$
\mathcal{R}_{p}=\left[\mathcal{R}_{s} /\left(2-\mathcal{R}_{s}\right)\right]^{2} .
$$

Figure 3 shows the unextinguished $s$ and $p$ intensity reflectances as functions of the substrate refractive index.

The intensities of the orthogonally polarized half-fields of the reflected light can be equalized if the incident light is (at least partially) linearly or elliptically polarized and its azimuth is adjusted with respect to the plane of incidence. The latter adjustment can be made by simply rotating the substrate (surface normal) without change of the (Brewster) angle of incidence.

The substrate should have as high a refractive index as possible, so that the film refractive index may assume reasonable (not-too-low) values, and the unextinguished reflectances could be as high as possible. Semiconductors are good candidates for this application. $\mathrm{GaP}$ is essentially transparent for wavelengths $>0.55 \mu \mathrm{m}$. For He-Ne laser light ( $\lambda$ $=6328 \AA$ ), it has a refractive index ${ }^{7} N_{2}=3.33$. If we assume that light is incident from air $\left(N_{0}=1\right)$, we find from Eq. (10b) that the required film refractive index $N_{1}=1.354$. A coating material with this index is cryolite. For operation as a DOWPBS and TFRHSD, the film thickness must be $1652 \AA$, from Eq. (11). Light must be incident at the Brewster angle, $73.28^{\circ}$, from Eq. (2). The unextinguished $s$ and $p$ intensity reflectances of the uncoated and coated areas are 69.65 and $28.55 \%$, respectively, as is obtained from Eqs. (12) and (13b).

As another case, consider the use of a Ge substrate $\left(N_{2}=\right.$ 4) coated by a film of the proper refractive index $N_{1}=1.372$ (e.g., $\mathrm{ThF}_{4}$ ) as an infrared DOWPBS and TFRHSD. In this case, the polarizing angle is $75.96^{\circ}$, and the unextinguished reflectances are $\mathcal{R}_{s}=77.85 \%$ and $\mathcal{R}_{p}=40.63 \%$. The required least film thickness $d_{s}=0.2577 \lambda=2.7316 \mu \mathrm{m}$ for $10.6-\mu \mathrm{m} \mathrm{CO}$ laser radiation.

Figure 4 shows two examples of coating patterns and the corresponding reflected polarization patterns.

For the $s$-polarizing angle $\phi_{s}$ to equal the $p$-polarizing angle 

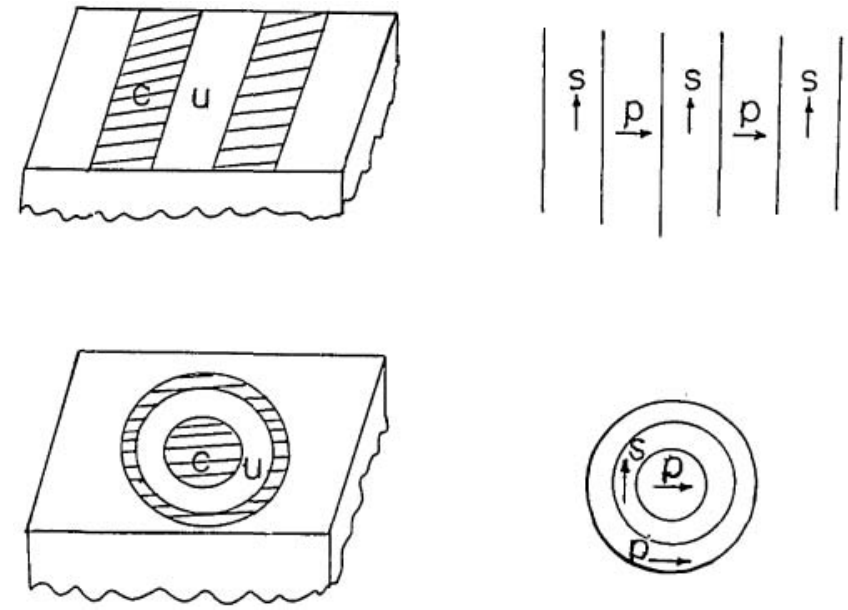

Fig. 4. Examples of thin-film coating patterns and corresponding reflected polarization patterns. $c$ and $u$ indicate the coated and uncoated areas of the substrate, respectively.

$\phi_{p}$, the (normalized) film refractive index $n_{1}$ must take the value given by Eq. (10). Because a refractive-index error is inevitable, it is important to consider its effect. The rate at which $\phi_{s}$ shifts as $n_{1}$ is changed is obtained by differentiation of Eq. (8) and use of the condition of Eq. (10); this gives

$$
\begin{gathered}
\frac{d \phi_{s}}{d n_{1}}=-\frac{n_{1}^{2}\left(2-n_{1}^{2}\right)^{1 / 2}}{\left(n_{1}^{2}-1\right)^{2}} \\
=-\frac{2 \sqrt{2} n_{2}^{2}\left(n_{2}^{2}+1\right)^{1 / 2}}{\left(n_{2}^{2}-1\right)^{2}} \\
\simeq-2 \sqrt{2} / n_{2} \text {, when } n_{2}^{2} \gg 1 .
\end{gathered}
$$

If we assume an air ambient $\left(N_{0}=1\right)$ and a Ge substrate $\left(N_{2}\right.$ $=n_{2}=4$ ), we get $d \phi_{s} / d n_{1}=-0.829 \mathrm{rad}$ per unit change of $n_{1}$. To bring $\phi_{s}$ to within $\pm 1^{\circ}$ of $\phi_{p}\left(75.96^{\circ}\right), n_{1}$ must be tuned to within \pm 0.02 of the required value (1.372). This is achievable using present day advanced and well-controlled vacuum evaporation technology and other film-formation techniques.
The effect of small film-thickness error is determined by the correspondingly small reflection coefficient for the $s$ polarization when $d$ deviates from the polarizing thickness $d_{s}$ [Eq. (11)] by a small amount. We express the fractional thickness shift by

$$
\Delta \eta=\left(d-d_{s}\right) / d_{s} .
$$

Differentiation of Eq. (3) with respect to $\zeta$, use of Eqs. (6), and some additional manipulations yield the following residual reflection coefficient and intensity reflectance:

$$
\begin{aligned}
& R_{s}=-j(\pi / 4)\left(n_{2}-\frac{1}{n_{2}}\right) \Delta \eta, \\
& \mathscr{R}_{s}=(\pi / 4)^{2}\left(n_{2}-\frac{1}{n_{2}}\right)^{2}(\Delta \eta)^{2} .
\end{aligned}
$$

If in the foregoing example of a Ge substrate $\left(n_{2}=4\right)$ we take $\Delta \eta=0.01$ (corresponding to $\Delta d=27.3 \mathrm{~nm}$ at $\lambda=10.6 \mu \mathrm{m}$ ), we obtain $\mathcal{R}_{s}=8.7 \times 10^{-4}$. This demonstrates very satisfactory tolerance to thickness errors, especially at longer wavelengths.

This work was supported by a grant from the State of Louisiana Board of Regents and the Foundation for A Better Louisiana.

\section{References}

1. Use of metallic substrates will be dealt with separately.

2. For a discussion of conventional division-of-amplitude polarizing beam splitters and half-shade devices see J. M. Bennett and H. E. Bennett, in Handbook of Optics, W. G. Driscoll and W. Vaughan, Eds. (McGraw-Hill, New York, 1978).

3. See, for example, R. M. A. Azzam and N. M. Bashara, Ellipsometry and Polarized Light (North-Holland, Amsterdam, 1977).

4. See, for example, E. Ritter, in Physics of Thin Films, Vol. 8, G. Hass, M. H. Francombe, and R. W. Hoffman, Eds. (Academic, New York, 1975).

5. H. K. Pulker, "Characterization of optical thin films," Appl. Opt. 18, 1969 (1979).

6. R. M. A. Azzam, "Direct relation between Freznel's interface reflection coefficients for the parallel and perpendicular polarizations," J. Opt. Soc. Am. 69, 1007 (1979).

7. D. E. Aspnes and A. A. Studna, "Dielectric Functions and Optical Parameters of $\mathrm{Si}, \mathrm{Ge}, \mathrm{GaP}, \mathrm{GaAs}, \mathrm{GaSb}, \ln \mathrm{P}, \ln \mathrm{As}$, and $\operatorname{lnSb}$ from 1.5 to $6.0 \mathrm{eV}$," Phys. Rev. B 27, 985 (1983).

\section{A REMINDER}

Authors submitting Rapid Communications for publication must remember to Include a letter from their Institution agreeing to honor the publication charge. Otherwise publication will be delayedand the whole idea of this section of Letters to the Editor is defeated. 